\title{
MADURACIÓN PULMONAR FETAL CON CORTICOIDES, UNA SITUACIÓN COMPLEJA EN LA MADRE CON DIABETES
}

\author{
FETAL LUNG MATURATION WITH CORTICOSTEROIDS, \\ A COMPLEX SITUATION IN THE MOTHER WITH DIABETES
}

Jorge Fabián Tedesco

\begin{abstract}
RESUMEN
La maduración pulmonar con corticoides fue un avance significativo en la lucha contra las complicaciones respiratorias que sufrían los niños prematuros, convirtiéndose en un hito en la historia de la Medicina moderna.

Se sabe que los hijos de madres con diabetes gestacional (DG) o prestacional (DPG) tienen una mayor incidencia de distrés respiratorio del recién nacido, por lo que se verían beneficiados con esta práctica médica. También se conoce el efecto hiperglucemiante de los corticoides que provocan una inhibición de la producción del surfactante. De esta manera no se obtendrían los efectos beneficiosos a nivel respiratorio, y además se pondría en riesgo tanto a la madre como al hijo. Por esto resulta fundamental el control metabólico estricto durante la maduración pulmonar con corticoides mediante la implementación de protocolos de monitoreo glucémico y de infusión de insulina para lograr los resultados deseados y evitar, o minimizar, los riesgos.
\end{abstract}

Revista de la Sociedad Argentina de Diabetes 2018; Vol. 52 (75-85)

\begin{abstract}
Lungs maturation with corticosteroids has been a very significant advance in the prevention of respiratory complications of premature infants, becoming a milestone in the history of modern medicine.

It is known that the children of mothers with gestational or pre-gestational diabetes have a higher incidence of respiratory distress syndrome of the newborn, so they are benefited by this medical practice. Strict metabolic control during this treatment is essential through the implementation of glycemic monitoring and insulin infusion protocols, since the hyperglycemic effect of corticosteroids could cause an inhibition of surfactant production, which would impede its effect on lung maturation not obtaining, consequently, the beneficial effects at the respiratory level. For this reason, different treatment schemes have been established in order to obtain the desired results, avoiding or minimizing the risks.
\end{abstract}

Revista de la Sociedad Argentina de Diabetes 2018; Vol. 52 (75-85)
Especialista Universitario en Diabetes egresado de la Universidad Nacional de Córdoba, especialista en Clínica Médica, miembro del Comité de Diabetes y Embarazo de la Sociedad Argentina de Diabetes, ex Instructor de la Residencia de Clínica Médica del Hospital San Martín, Médico de Planta del Servicio de Endocrinología, Diabetes y Nutrición del Hospital San Martín, Paraná, Entre Ríos
Contacto de la autor: Jorge Fabián Tedesco

E-mail: tedescofabian@yahoo.com.ar

Correspondencia: Gregoria Matorras de San Martín 890,

Paraná, Entre Ríos, Argentina

Fecha de trabajo recibido: 06/08/18

Fecha de trabajo aceptado: 11/01/19

Conflictos de interés: el autor declara que no existe conflicto de interés

\section{Maduración pulmonar fetal y diabetes materna Introducción}

Durante la vida intrauterina el aparato respiratorio es uno de los últimos en madurar, tanto estructural como funcionalmente, siendo su desarrollo completo imprescindible para la adaptación a la vida extrauterina. Dentro de este proceso es de fundamental importancia la aparición del surfactante pulmonar; esto marca la diferencia entre feto inmaduro y prematuro, y es por lo tanto un hito en el crecimiento fetal'.

El desarrollo pulmonar ocurre como una serie de eventos dinámicos que se relacionan estrechamente entre sí. A efectos de su comprensión, se han descrito varias etapas:

- Etapa embrionaria (3 a 7 semanas): desarrollo de vías aéreas mayores, formación de los bronquios principales.

- Etapa pseudoglandular (7 a 17 semanas): desarrollo del árbol bronquial hasta nivel de bron- 
quíolos terminales (preacinar). Comienza el desarrollo de la circulación pulmonar (vasculogénesis).

- Etapa canalicular (17-27 semanas): transición a un potencial pulmón viable. Se forman los bronquiolos respiratorios y los conductos alveolares. Crecimiento del lecho capilar (angiogénesis). Después de 20 semanas de gestación, las células epiteliales cúbicas comienzan a diferenciarse en células alveolares de tipo II con la formación de surfactante que se almacena en los cuerpos lamelares citoplasmáticos.

- Etapa sacular (28-36 semanas): continúa la división de la vía aérea periférica. Crece la superficie de intercambio gaseoso. Los neumocitos tipo II aumentan el número de cuerpos lamelares. Los alvéolos comienzan a aparecer después de las 30 semanas.

- Etapa alveolar (36 semanas a 2-3 años postnatal): formación de alvéolos. Marcada proliferación de todos los tipos celulares. Continúa el proceso de angiogénesis ${ }^{2}$.

Durante el tercer trimestre del embarazo el pulmón fetal se llena de surfactante, un fluido tensoactivo producido por los neumocitos tipo II que actúa luego del nacimiento reduciendo la tensión superficial en la interface aire-líquido para facilitar así la expansión pulmonar y evitar el colapso alveolar. El surfactante pulmonar es una mezcla compleja que se compone de un $90 \%$ de lípidos, principalmente fosfolípidos, y cerca de un 10\% de proteínas; su déficit es el principal mecanismo responsable del síndrome de dificultad respiratoria (SDR).

En el pulmón con actividad surfactante inadecuada se produce mayor tensión superficial, lo que conduce a colapso alveolar, bajo volumen respiratorio y disminución de la compliance, con la consiguiente hipoxemia, siendo una de las principales causas de morbimortalidad neonatal.

Debido a movimientos respiratorios y deglutorios fetales, los componentes del surfactante se detectan en el líquido amniótico durante el tercer trimestre; esto permite realizar su dosaje y predecir la madurez pulmonar.

Se cuentan con diferentes pruebas basadas en la medición de dichos componentes. Algunas son:

- Índice lecitina/esfingomielina (L/E): predice bajo riesgo de desarrollo del SDR cuando es >a 2.

- Medición de fosfatidilglicerol (FG): su presencia indica un estado avanzado de desarrollo pulmonar. Puede expresarse cualitativamente como positivo o negativo, o de manera cuantitativa. Si es positivo se asocia a una baja tasa de dificultad respiratoria.
- Índice surfactante/albumina: los valores superiores a $55 \mathrm{mg} / \mathrm{g}$ se consideran maduros, inferiores a $40 \mathrm{mg} / \mathrm{g}$ inmaduros y los valores de 40 a $54 \mathrm{mg} / \mathrm{g}$ se establecen como "indeterminados".

- Recuento de cuerpos lamelares: es una medida directa de la producción de surfactante por los neumocitos de tipo II.

Todas las pruebas anteriores son equiparables y evalúan en forma indirecta la madurez pulmonar y miden la probabilidad de desarrollo del SDR ${ }^{3}$.

Su determinación es de utilidad cuando se planea finalizar el embarazo de manera electiva y la fecha exacta de gestación es incierta. No está indicada su realización en embarazos con edad gestacional bien documentada igual o mayor a 39 semanas (madurez pulmonar muy probable) o menor a 32 semanas (inmadurez pulmonar muy probable).

Luego de la publicación de datos controversiales respecto de la utilidad de marcadores bioquímicos en embarazadas con diabetes mellitus (DM), principalmente en relación al del índice L/E, el consenso actual es que el valor umbral en las pruebas de madurez pulmonar puede utilizarse tanto en embarazadas sin o con diabetes, tanto pregestacional (DPG) como gestacional (DG)

Con el objetivo de evaluar el momento adecuado para finalizar el embarazo, la Federación Argentina de Sociedades de Ginecología y Obstetricia (FASGO) sugiere investigar la madurez pulmonar en toda paciente con diabetes en la que existan dudas sobre la edad gestacional, como en caso de fecha de última menstruación (FUM) incierta y ecografía tardía, siendo recomendable, en este caso, determinar FG y/o índice L/E ${ }^{6}$.

En pacientes con buen control glucémico la indicación es igual que para pacientes sin diabetes dado que el riesgo de SDR es similar. En aquellas con mal control glucémico se aconseja su realización si el parto se contempla antes de las 38 semanas $^{3}$.

Esto se debe a que, dentro de las complicaciones neonatales en hijos de madres con diabetes, el retraso en la maduración pulmonar y la probabilidad de presentar SDR es un hecho documentado?.

Los fetos de embarazos complicados con DM retrasan su maduración pulmonar en aproximadamente 1,5 semanas respecto de embarazos sin DM, medido por la aparición tardía de indicadores bioquímicos de madurez ( $F G$ y la relación L/E) ${ }^{8}$ (Figura 1).

Al analizar gestaciones con DM consecutivas, documentando el control de la glucemia materna y la presencia de FG, Piper et al. demostraron 
que el control metabólico durante el embarazo tiene consecuencias positivas sobre el desarroIlo pulmonar9; estos autores definieron el control glucémico como "bueno" si la glucosa media en sangre era $<105 \mathrm{mg} / \mathrm{dl}$ y pobre si era mayor. Se analizó un total de 621 embarazos con diabetes, 261 con buen control y 360 con mal control; el FG estuvo ausente en el $21 \%$ del grupo de buen control, frente al $31 \%$ de los embarazos con mal control $(p<0,05)$. Cuando se estratificó por la edad gestacional, el riesgo de ausencia de FG fue significativamente mayor en el grupo de mal control glucémico (OR 1,83, IC95\% 1,19 a 2,84).

Todos los casos de enfermedad de membrana hialina más allá de las 32 semanas se produjeron en embarazos con pobre control glucémico, sin haber casos de SDR luego de las 37 semanas de gestación (Figura 2) ${ }^{10}$. Los autores concluyeron que los niveles de glucosa materna mal controlados se asocian con aparición retardada de FG.

El control metabólico durante el embarazo complicado con DM, ya sea gestacional o pregestacional, es fundamental para disminuir la morbimortalidad neonatal. Conseguir los objetivos glucémicos mejora los resultados de indicadores bioquímicos de madurez pulmonar y los hace equiparables al de pacientes sin $\mathrm{DM}^{11}$.
Si bien se sabe que la hiperglucemia materna, y por ende fetal, provoca hiperplasia de células beta en el páncreas del feto con el consiguiente hiperinsulinismo, el mecanismo fisiopatológico por el cual la diabetes retarda el desarrollo pulmonar fetal no está completamente establecido; dichas alteraciones, hiperglucemia e hiperinsulinismo fetal, son los principales mecanismos involucrados en la menor producción de surfactante. A nivel celular se desarrolla un mecanismo complejo y se producen interacciones epitelio-mesénquima por medio de mediadores bioquímicos y hormonales.

Estudios experimentales en pulmón de rata evidenciaron que el cortisol actúa a nivel de fibroblastos pulmonares al estimular la síntesis de un polipéptido de bajo peso molecular, el factor pneumocito-fibroblasto (FPF), el cual induce la síntesis de surfactante a nivel de los neumocitos tipo II $^{12}$.

En desarrollos de cultivos celulares mixtos fibroblastos-pneumocitos, en medios enriquecidos con insulina, se bloquea el estímulo del cortisol sobre la síntesis de FPF inhibiendo el desarrollo del surfactante. Dicho efecto inhibitorio de la insulina no se demuestra en cultivos mono-celulares de neumocitos II estimulados por FPF, por lo cual el efecto antagónico de la insulina sobre el cortisol se encontraría presumiblemente a nivel de fibroblastos ${ }^{12}$.

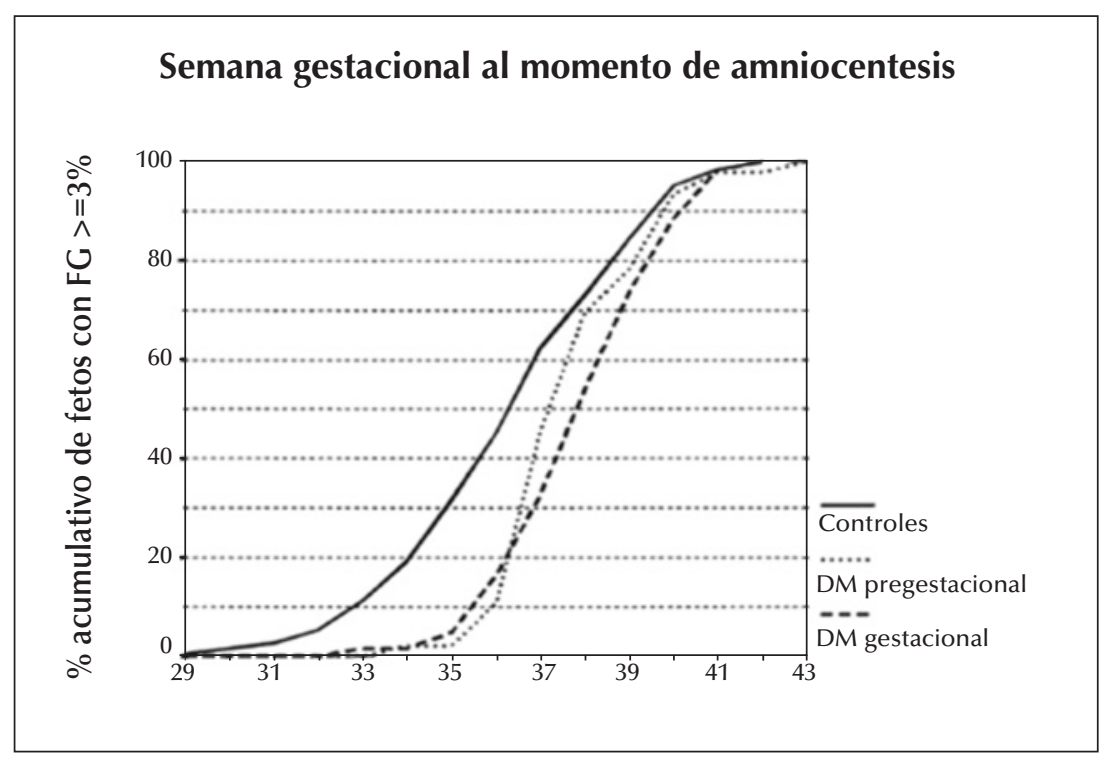

Fuente: adaptado de Moore TR. A comparasion of amniotic fluid fetal pulmonary phospholipids in normal and diabetic pregnancy. Am J Obst Gynecol 2002.

Figura 1: Porcentaje acumulativo de fetos con $\mathrm{FG}>=3 \%$ durante el tercer trimestre estratificado por el tipo de diabetes. 


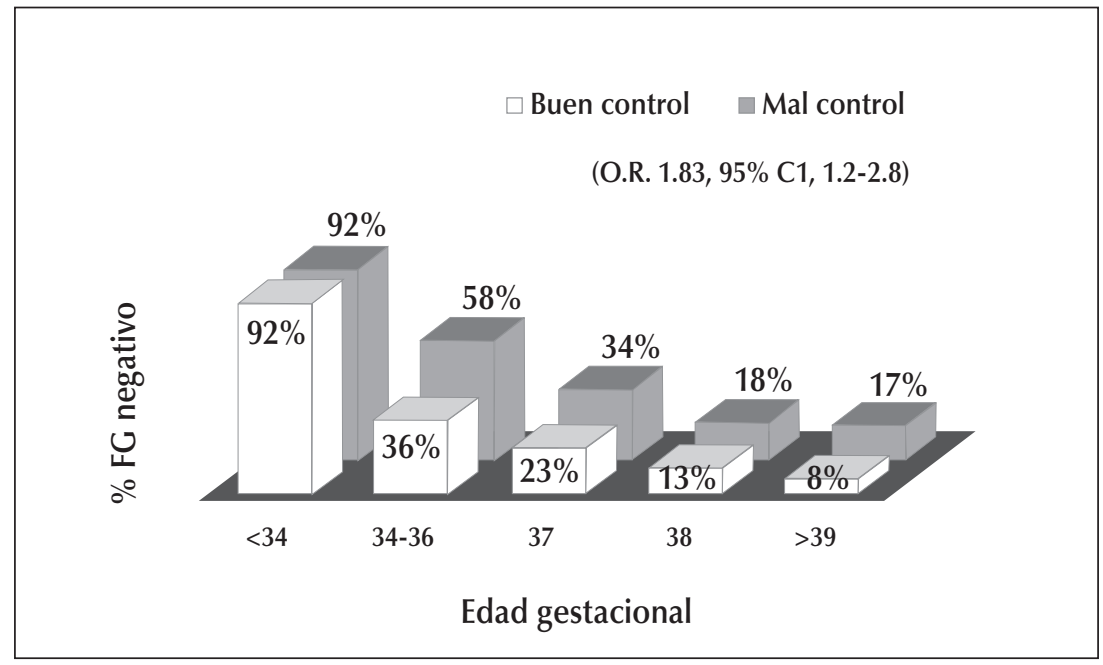

Fuente: adaptado de Piper JM. Delayed appearance of pulmonary maturation markers is associated with poor glucose control in diabetic pregnancies. The Journal of Maternal Fetal Medicine 1998; 7:148-153.

Figura 2: Maduración pulmonar en pacientes diabéticas.

\section{Maduración pulmonar con glucocorticoides}

Desde los primeros trabajos de Sir Graham Liggins, en Nueva Zelanda, se conocen los beneficios de la maduración pulmonar con corticoides. Esta práctica médica tiene indicaciones precisas para evitar la mala utilización de la misma:

1. Toda mujer con riesgo de parto prematuro que se encuentre entre las 24 y 34 (+6 días) semanas de gestación (A) (ver Anexos 1 y 2).

2. Los corticoides prenatales deben darse a todas las mujeres para las que se prevé una cesárea electiva antes de las 38 (+6 días) semanas de gestación (A).

3. En gestaciones de más de 34 semanas cuando hay evidencia de inmadurez pulmonar (B).

4. Podría ser considerada para los embarazos entre las $23(0)$ y 23 (+6 días) semanas de gestación con riesgo de parto prematuro (C).

Las indicaciones 1 y 2 tienen el mejor nivel de evidencia ${ }^{13-14}$. Una revisión realizada en $2017^{15}$ para la Librería Cochrane muestra que una dosis de corticoides prenatales en mujeres con riesgo de parto prematuro produce una reducción global y estadísticamente significativa (las primeras $48 \mathrm{~h}$ ) en muerte peri y neonatal, distrés respiratorio, hemorragia intraventricular, enterocolitis necrotizante, necesidad de ventilación mecánica e infecciones sistémicas (A).
Posteriormente a la maduración pulmonar, la recomendación con mayor evidencia es realizar el parto o cesárea después de las $24 \mathrm{~h}$ y antes de los siete días $(A)^{16}$.

Si bien se han utilizado múltiples tipos de corticoides con diferentes vías de administración de los mismos, en la actualidad los de mayor evidencia son betametasona y dexametasona por vía intramuscular (A). La dosis de betametasona es de $12 \mathrm{mg}$ IM cada $24 \mathrm{~h}$, un total de dos dosis, y la dexametasona $6 \mathrm{mg}$ cada $12 \mathrm{~h}$, por un total de cuatro dosis ${ }^{17}$.

Al momento de elegir uno de estos corticoides hay que tener en cuenta que la revisión de Cochrane sobre los corticoides prenatales para acelerar la maduración pulmonar fetal en mujeres con riesgo de parto prematuro sugiere que el tratamiento con betametasona provoca una mayor reducción del SDR que la dexametasona $(1++)^{16}$. A favor de la dexametasona podría afirmarse que disminuyó la incidencia de hemorragia intraventricular comparada con betametasona (OR 0,44, IC95\%: 0,21 a 0,92; cuatro ensayos, 549 neonatos) $(1++)^{17}$.

Otro estudio de cohortes históricas utilizó el análisis de regresión logística multivariada para comparar los dos grupos tratados con esteroides entre sí. Demostró que el riesgo de muerte neonatal fue menor con betametasona (OR 0,44; IC95\%: 0,29-0,68) que con dexametasona (OR 0,73; IC95\%: 0,47-1,14) $(p<0,05)(2-)^{18}$. 
Un gran estudio retrospectivo no aleatorizado sugirió que los bebés expuestos a la betametasona antenatal tienen menos leucomalacia periventricular quística neonatal que los bebés expuestos a dexametasona (2-) $)^{19}$.

También podría agregarse que la betametasona es el corticoide más utilizado en el mundo para este fin, además de ser el que se empleó en los primeros estudios de Sir Grahan Liggins.

Ciclos repetidos semanales de corticoides prenatales reducen la incidencia y la gravedad de la enfermedad respiratoria neonatal, pero los beneficios a corto plazo se asocian con una reducción en el peso y el perímetro cefálico. No se recomiendan los ciclos repetidos semanales (A). Un ciclo de rescate sólo debería considerarse con precaución en aquellos embarazos que recibieron uno antes de las 26 semanas y otra indicación obstétrica surge más tarde en el embarazo (D) ${ }^{14}$.

De todo esto y según la evidencia actual, surge una pregunta: ¿cuál es la fuerza de la recomendación para el uso de corticoides en la paciente complicada con diabetes gestacional o diabetes previa? Es muy difícil de formular una respuesta dado que en todos los ensayos clínicos las pacientes con estas complicaciones fueron excluidas de los mismos.

\section{Efecto de la maduración pulmonar con glucocorticoides sobre la glucemia materna}

Según la Sociedad Argentina de Diabetes (SAD) y la Asociación Latinoamericana de Diabetes (ALAD), los objetivos glucémicos son $90 \mathrm{mg} / \mathrm{dl}$ en ayuno, y 140 y $120 \mathrm{mg} / \mathrm{dl}$ a los 60 y 120 minutos respectivamente. Estos objetivos se deben cumplir, con o sin la administración de corticoides, tratando de no tener más de un $20 \%$ de los mismos por fuera de lo esperado.

Los glucocorticoides afectan el metabolismo de la glucosa en forma contraria a los efectos de la insulina, inhiben la captación de glucosa en los tejidos periféricos (tejido muscular y adiposo), mientras que promueven la liberación hepática de glucosa mediante gluconeogénesis.

También existe inhibición de la secreción de insulina, lo que contribuye en parte al desarrollo de hiperglucemia.

Ambos efectos son dosis-dependientes, a más dosis, más resistencia a la insulina y mayor inhibición de la secreción de la misma.

El patrón de la hiperglucemia inducida por los glucocorticoides se caracteriza por una hiperglucemia postprandial muy marcada y un efecto menor sobre la glucemia basal.

El grado de hiperglucemia se correlaciona con el grado de intolerancia a la glucosa preexistente.

El embarazo es un estado de resistencia a la insulina y el tratamiento con glucocorticoides para acelerar la maduración pulmonar fetal tiene consecuencias adversas sobre la glucemia materna. Esto es evidente incluso en mujeres sin diabetes previa o gestacional ${ }^{20}$.

Matrobattista et al. estudiaron el efecto del uso de betametasona sobre la glucemia a los 60 minutos luego de la sobrecarga oral con $50 \mathrm{~g}$ de glucosa (Glucose Challenge Test) en embarazadas sin diabetes con riesgo de parto prematuro y la duración de su efecto (Tabla 1). Los resultados indicaron que los efectos hiperglucemiantes de los corticoides son transitorios, siendo razonable posponer la prueba de tolerancia oral a la glucosa en las mujeres embarazadas que fueron sometidas a este tratamiento, al menos una semana, debido a los resultados falsos positivos que estos fármacos pueden provocar ${ }^{21}$.

Otros estudios arrojaron resultados similares. En los embarazos gemelares, tal vez debido a la mayor masa placentaria, se detectaron mayores niveles de insulina y hormonas de contrarregulación, tales como glucocorticoides, lactógeno placentario, progesterona, estrógenos y otros, aumentando el riesgo de intolerancia a la glucosa. La administración de corticoides en dichos embarazos aumenta el estado de resistencia a la insulina y provoca cifras de glucemia mayores que en embarazos no gemelares en madres sin diabetes ${ }^{22,23}$.

El riesgo de parto pretérmino aumenta en embarazos complicados con diabetes, ya sea espontáneo o por indicación médica, siendo el uso de glucocorticoides una práctica frecuente en este grupo de pacientes.

En las pacientes sin diabetes se produce una elevación leve y transitoria de la glucemia, a diferencia de esto, en las pacientes con diabetes el aumento en la glucemia puede ser hasta un $50 \%$ mayor con consecuencias directas sobre la madre y el feto. Aunque poco frecuente, se han descripto casos de cetoacidosis diabética ${ }^{24,25}$.

Durante la hiperglucemia materna se produce un aumento de la glucemia fetal con el consiguiente hiperinsulinismo, lo cual puede provocar hipoglucemia neonatal y bloquear la acción esti- 
mulante de los esteroides sobre la síntesis del surfactante pulmonar perdiendo los beneficios del tratamiento indicado 26,27 .

Es lógico suponer que durante la inducción de la madurez pulmonar con glucocorticoides deban modificarse los controles y el tratamiento de la hiperglucemia. Existen pocos estudios que definan el manejo preciso de la misma, y los existentes se realizaron con escaso número de pacientes. Esto puede deberse al grupo heterogéneo de pacientes embarazadas complicadas con diabetes, ya sea con diabetes previa al embarazo, tipo 1 ó tipo 2, con diabetes gestacional, con o sin requerimiento previo de insulina.

En cuanto a las madres con DG y DM2, Ramírez-Torres et al. analizaron el impacto del tratamiento con betametasona sobre la glucemia ${ }^{28}$. Esta cohorte analizó cuatro grupos de embarazadas con 10 pacientes cada uno:

- Grupo 1: sin DM.

- Grupo 2: DG sin requerimiento de insulina.

- Grupo 3: DG con requerimiento de insulina.

- Grupo 4: con DM2 con o sin requerimiento de insulina.

Las pacientes fueron internadas por cuatro a cinco días, se aumentaron los controles glucémicos antes y después de cada alimento y se ajustó la dosis de insulina para lograr objetivos en ayuno menor a $95 \mathrm{mg} / \mathrm{dL}$ y $2 \mathrm{~h}$ postprandial menor a $120 \mathrm{mg} / \mathrm{dL}$.

El grupo de embarazadas control presentó elevaciones discretas sin requerimiento de intervención médica.

La glucemia capilar de las embarazadas con diabetes se elevó de forma progresiva y significativa.

Cuatro pacientes del grupo 2 requirieron insulina, con una dosis de 0,3 U/kg/día calculada con el peso ideal sin embarazo.

Las mujeres del grupo 3 requirieron aumentos de $39,4 \%$ en el día 2 , de $67 \%$ en el día 3 , de $112,8 \%$ en el día 4 y de $72,3 \%$ en el día 5 respecto de la dosis del primer día.

Las pacientes del grupo 4 requirieron incrementos diarios de $26,4 \%$ en el día 2 , de $50,6 \%$ en el día 3, de $41 \%$ en el día 4 y de $64 \%$ en el día 5.

Además se realizó la toma de muestra para medición de $\mathrm{HbA} 1 \mathrm{c}$ en el momento previo a la colocación de la betametasona y dos semanas después. Se evidenció un aumento de la $\mathrm{HbA} 1 \mathrm{c}$ en los grupos de DG y DM2, demostrando un aumento en el promedio glucémico posteriormente a la colocación del corticoide. Es importante aclarar que la betametasona utilizada no era de depósito.

Otro estudio de monitoreo continuo ${ }^{29}$ en pacientes con DG $(n=6)$ y pacientes $\sin D M(n=3)$ mostró un mayor promedio glucémico en las pacientes con diabetes luego de la colocación de la betametasona intramuscular pero sin diferencia estadísticamente significativa. Este resultado podría deberse al escaso número de pacientes del estudio. Sí se evidenció una mayor variabilidad glucémica en el grupo de pacientes con diabetes con picos que alcanzaron valores de $370 \mathrm{mg} \%$.

\begin{tabular}{|c|c|c|c|}
\hline Paciente N & Control (mg/dl) & Día 1 (mg/dl)* & Día 3 (mg/dl) \\
\hline 1 & 120 & 143 & 109 \\
\hline 2 & 115 & 136 & 114 \\
\hline 3 & 104 & 145 & 145 \\
\hline 4 & 130 & 157 & 140 \\
\hline 5 & 109 & 153 & 155 \\
\hline 6 & 103 & 163 & 136 \\
\hline 7 & 120 & 149 & 106 \\
\hline
\end{tabular}

Control significa valores de glucemia 7-10 días antes de la betametasona. Día 1 significa 24 h después de la segunda betametasona. Día 3 significa 72 h después. * Los valores del día 1 fueron estadísticamente diferentes al control (p:0,03). Adaptado de: Mastrobattista JM, et al. Betamethasone alteration of de one-hour glucose challenge test in pregnancy. $J$ Reprod Med 2001; 46:83-86.

Tabla 1: Efecto de la betametasona en la curva de la tolerancia oral a la glucosa de $1 \mathrm{~h}$ con $50 \mathrm{~g}$ de glucosa. 


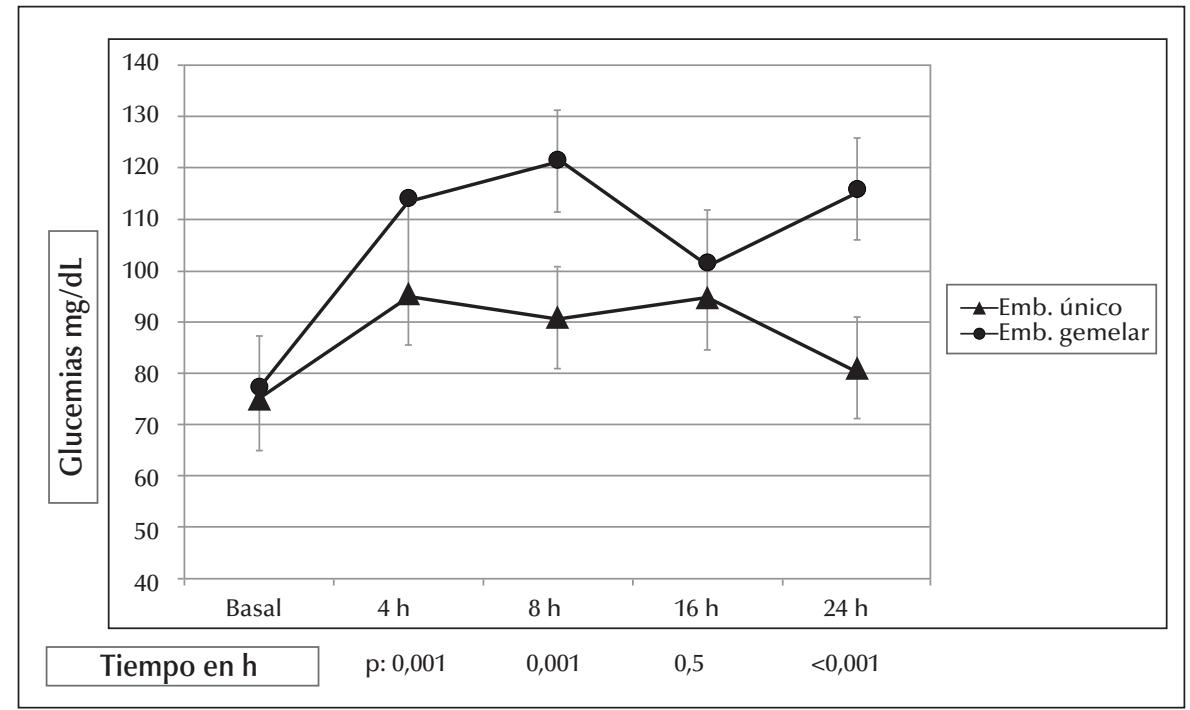

Fuente: adaptado de Foglia LM, Deering SH, Lim E, et al. Maternal glucose levels after dexamethasone for fetal lung development in twin vs singleton pregnancies. Am J Obstet Gynecol 2008 Oct; 199 (4):380.

Figura 3: Niveles de glucosa materna después de la administración de dexametasona para la maduración del pulmón fetal en embarazos gemelares vs embarazos únicos.

\section{Tratamiento. Generalidades}

La DM no es una contraindicación para el uso de glucocorticoides; las mujeres con DPG o DG que reciben esta medicación para la maduración pulmonar fetal deben recibir insulina según un protocolo previamente establecido y un monitoreo glucémico estricto ${ }^{30}$. Las recomendaciones ${ }^{31-32}$ que deberían cumplirse son:

1. Paciente internada.

2. Estricto control del medio interno materno.

3. Goteo IV insulina con bomba.

4. Objetivo preprandial $<90 \mathrm{mg} / \mathrm{dl}$ y postprandial $<120 \mathrm{mg} / \mathrm{dl}$.

5. Ausencia de hipoglucemias.

6. Controles de glucemia capilar horaria.

7. Aportes cuidadosos de comidas y colaciones.

8. Estricto control clínico.

9. Laboratorio.

10. Estricta vigilancia de salud fetal.
Manejo de infusión continua de insulina

En el consenso de la SAD, en su recomendación para el manejo de las madres con diabetes DPG publicado en $2009^{31}$, se hace referencia a que la cetoacidosis es una complicación poco frecuente pero grave, con una mortalidad fetal entre el 20 y $35 \%$ y una incidencia de $1,73 \%$ en DPG y $0,7 \%$ en DG, pudiendo ser desencadenada por el uso de glucocorticoides. Por lo tanto, recomienda extremar los cuidados durante su realización, siendo la descompensación metabólica una contraindicación para la misma.

Por lo expuesto, se debe internar a la paciente y suspender la insulina habitual, utilizar corticoides que no sean de depósito y aumentar los controles glucémicos a uno por hora con el fin de mantener la glucemia entre 80 y 120 mg/dl. Existen múltiples esquemas de infusión durante la maduración pulmonar, a continuación se describen algunos de ellos. 


\section{Iniciar goteo a razón de 0,5 - 1,5 UI/h}

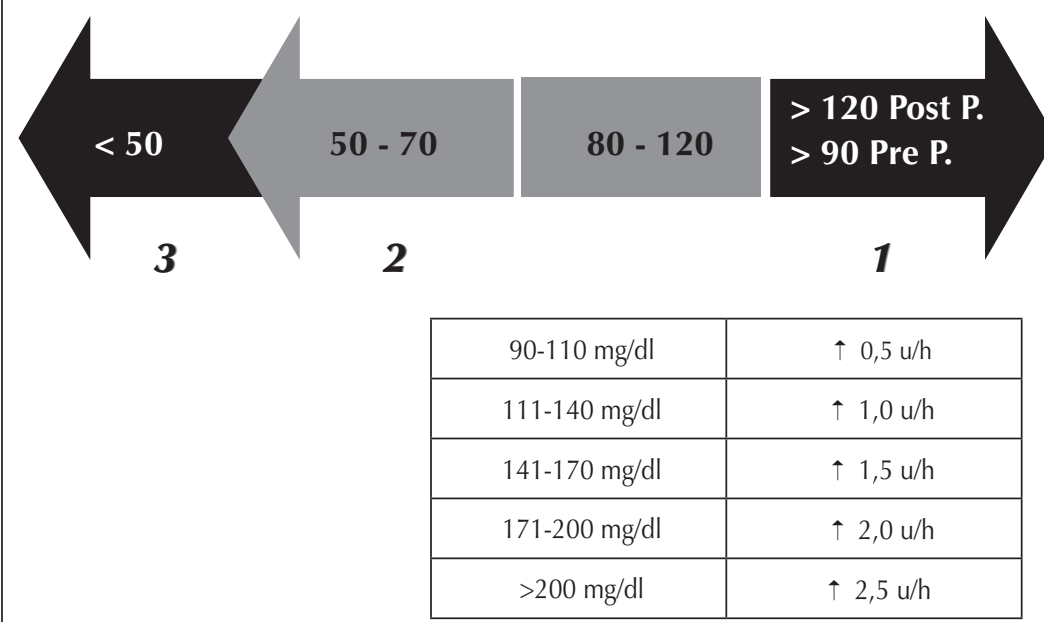

1. Aumentar el goteo de insulina.

2. Disminuir el goteo entre 0,5 y $1 \mathrm{Ul} / \mathrm{h}$.

3. Cerrar el goteo, controlar cada 15 minutos y cuando llegue a $90 \mathrm{mg} / \mathrm{dl}$, iniciar el goteo de con $1 \mathrm{Ul} / \mathrm{h}$ menos.

Antes de comenzar a almorzar, merendar o cenar, aumentar durante 2 h, 2-3 UI/hora la infusión continua de insulina, desde 15 minutos antes de comenzar a comer ${ }^{31-32}$.

Tabla 2: Ejemplo de aumento de la dosis de insulina según control de glucosa capilar.

Con el objetivo de contrarrestar el efecto hiperglucemiante de los glucocorticoides, existen estudios publicados que analizan cómo incrementar la dosis de insulina en pacientes embarazadas con DM1.

Un algoritmo propuesto es el aumento de un $40 \%$ del total de la dosis de insulina subcutánea (basal y bolo) previa a la colocación de la primera dosis de corticoide intramuscular intentado contrarrestar el aumento glucémico que éstos producen ${ }^{33}$.

El porcentaje de aumento se estimó luego de analizar la glucemia y la dosis de insulina rápida requerida para normalizar las cifras obtenidas en una cohorte de mujeres sometidas al tratamiento con betametasona con objetivos en ayuno de 70 a $110 \mathrm{mg} / \mathrm{dl}(4-6 \mathrm{mmol} / \mathrm{l})$ y postprandial menos de $140 \mathrm{mg} / \mathrm{dl}(8 \mathrm{mmol} / \mathrm{l})$.

La dosis diaria de insulina en la cohorte sometida al algoritmo de corrección (segunda) se aplicó desde la primera noche del tratamiento con betametasona según los siguientes valores:

- Día 1: aumento del $25 \%$ del total de la dosis de insulina previa al corticoide.

- Día 2: aumento del $40 \%$ de la dosis previa al corticoide.

- Día 3: aumento del $40 \%$ de la dosis previa al corticoide.

- Día 4: aumento del $20 \%$ de la dosis previa al corticoide.

- Día 5: aumento del 10-20\% de la dosis previa al corticoide.
- Día 6-7: disminución gradual de la dosis.

La glucemia media durante el segundo y tercer día se redujo significativamente en comparación con la primera cohorte $(p<0,05)$ (Tabla 3$)$, y se encontraba en niveles aceptables.

A pesar de ello todas las pacientes de la segunda cohorte recibieron dosis extra de insulina rápida. Ninguna de las pacientes presentó episodios de hipoglucemia.

Este estudio demuestra que es posible lograr un control metabólico aceptable en mujeres con DM1 mediante el aumento de la dosis subcutánea de insulina en aproximadamente un $40 \%$ de su basal, luego de la inyección de glucocorticoides y antes de la elevación de la glucemia.

Ante la evidente necesidad de un ajuste en el tratamiento de pacientes diabéticas para el manejo durante la maduración pulmonar, es posible individualizar los requerimientos extras según la dosis previa de insulina, antes de la administración de corticoides, tanto en DG como DPG ${ }^{34}$. Se mantiene el esquema subcutáneo habitual, y se administra por infusión endovenosa continua, insulina suplementaria a una dosis determinada según el requerimiento total diario. La infusión endovenosa se inicia inmediatamente antes de la inyección de corticoides, se ajusta según medición horaria de glucemia capilar, y se detiene $12 \mathrm{~h}$ luego de la última aplicación de esteroides (Tabla 4). Si los 
valores glucémicos exceden el límite de $180 \mathrm{mg} / \mathrm{dl}$ en dos mediciones horarias consecutivas, la dosis se escala al régimen siguiente, y si son inferiores a $108 \mathrm{mg} / \mathrm{dl}$ se desciende al régimen previo.

\begin{tabular}{|c|c|c|c|c|}
\hline & \multicolumn{2}{|c|}{$\begin{array}{c}\text { Dosis de insulina } \\
\text { (\% de incremento) }\end{array}$} & \multicolumn{2}{c|}{ Glucemia (mg/dl) } \\
\hline & $\begin{array}{c}\text { Antes del } \\
\text { algoritmo }\end{array}$ & $\begin{array}{c}\text { Después del } \\
\text { algoritmo }\end{array}$ & $\begin{array}{c}\text { Antes del } \\
\text { algoritmo }\end{array}$ & $\begin{array}{c}\text { Después del } \\
\text { algoritmo }\end{array}$ \\
\hline Día 1\# & 6 & $27^{*}$ & 121 & 139 \\
\hline Día 2\# & 38 & 45 & 257 & $148^{*}$ \\
\hline Día 3 & 36 & 40 & 221 & $173^{*}$ \\
\hline Día 4 & 27 & 31 & 139 & 126 \\
\hline Día 5 & 17 & 11 & 139 & 133 \\
\hline
\end{tabular}

* $p<0,05$ comparada con primer cohorte.

\# dosis de betametasona, $12 \mathrm{mg}$ por la tarde.

Adaptado de Mathiesen ER, et al. Insulin dose during glucocorticoid treatment for fetal lung maturation in diabetic pregnancy: test of an algorithm [correction of analgoritm]. Acta Obstet Gynecol Scand 2002 Sep; 81(9):835-9.

Tabla 3: Glucemia y dosis de insulina en embarazadas con DM1 antes y después de un algoritmo de aumento de dosis de insulina durante la maduración pulmonar con corticoides.

\begin{tabular}{|l|c|c|c|c|}
\hline $\begin{array}{c}\text { Glucemia } \\
\text { horaria }\end{array}$ & \multicolumn{5}{|c|}{$\begin{array}{c}\text { Infusión EV de insulina (UI/H) } \\
\text { (según requerimiento previo de insulina }\end{array}$} \\
\hline & $\mathrm{A}(<40 \mathrm{U} / \mathrm{d})$ & $\mathrm{B}(40-80 \mathrm{U} / \mathrm{d})$ & $\mathrm{C}(81-120 \mathrm{U} / \mathrm{d})$ & $\mathrm{D}(>120 \mathrm{U} / \mathrm{d})$ \\
\hline 108 & 0 & 0 & 0 & 0 \\
\hline $108-126$ & 0,5 & 1 & 2 & 3 \\
\hline $127-144$ & 1 & 2 & 3 & 5 \\
\hline $145-162$ & 1,5 & 3 & 4 & 7 \\
\hline $163-180$ & 1 & 4 & 6 & 10 \\
\hline$>180$ & 3 & 6 & 8 & 13 \\
\hline
\end{tabular}

Fuente: adaptado de Kaushal K, Gibson JM, Railton A, et al. A protocol for improved glycaemic control following corticosteroid therapy in diabetic pregnancies. Diabet Med 2003 Jan; 20 (1):73-5.

Tabla 4: Dosis de inicio y ajuste de insulina endovenosa (EV) según requerimiento diario habitual y control glucémico horario.

\section{Estudios a largo plazo}

Se realizaron múltiples estudios de seguimiento sobre la población original incluida en los ensayos de Sir Grahan Liguins, con el objeto de evaluar el impacto a largo plazo en los hijos del tratamiento instituido a las gestantes, y se obtuvieron las siguientes conclusiones respecto de la exposición prenatal a betametasona para la prevención del SDR:

1. No altera la presión arterial (6 años de seguimiento) $)^{35}$.

2. No altera la función pulmonar o la prevalencia de sibilancias y asma (30 años de seguimiento) ${ }^{36}$.

3. Puede dar lugar a insulinoresistencia en la vida adulta, pero no tiene ningún efecto clínico sobre los factores de riesgo cardiovascular (30 años de seguimiento) ${ }^{37}$.

4. No altera el funcionamiento cognitivo, la memoria de trabajo y atención, la morbilidad psiquiátrica, la motricidad manual o la calidad de vida relacionada con la salud (31 años de seguimiento) $)^{38}$.

5. No afecta la masa ósea máxima o la estructura del fémur (31 años de seguimiento) ${ }^{39}$.

\section{CONCLUSIONES}

En base a la evidencia actual, la cual es muy escasa en este tema, se concluye que:

- La maduración pulmonar fetal con corticoides es necesaria, ante indicaciones precisas, para disminuir las complicaciones respiratorias en los niños prematuros.

- El control glucémico estricto es fundamental para conseguir una maduración pulmonar exitosa y evitar así complicaciones maternas y fetales.

- Deberían realizarse más estudios, sobre maduración pulmonar en las pacientes con diabetes previa o con diabetes gestacional, para sustentar su indicación en evidencia de mejor calidad.

\section{AGRADECIMIENTOS}

Al Dr. Víctor Franciso Commendatore y la Dra. Silvia Gorbán de Lapertosa.

\section{BIBLIOGRAFÍA}

1. Schwarcz RL, Duverges CA, Díaz AG, et al. Obstetricia. $6^{\text {ta }}$. Edición. Buenos Aires: Editorial El Ateneo, 2005; cap. 2:24-25.

2. Iñiguez F, Sánchez I. Desarrollo pulmonar. Revista Neumonología Pediátrica, Sociedad Chilena de Neumología Pediátrica 2008; Vol 3, 2:148-155.

3. Fetal lung maturity. ACOG Practice Bulletin N 97. American College of Obstetricians and Gynecologist. Obstet Gynecol 2008; 112:117-26. 
4. Delgado J, Greene M, Winkelman J, et al. Comparison of disaturated phosphatidylcholine and fetal lung maturity surfactant/ albumin ratio in diabetic and nondiabetic pregnancies. Am J Clin Pathol 2000; 113:233-239.

5. Melanson SE, Jarolim P, Mc Elrath TF, et al. Fetal lung maturity testing in diabetic mothers. Lab Med 2007; 38:553.

6. Consenso de Diabetes. Recopilación, actualización y recomendaciones para el diagnóstico y tratamiento de la diabetes gestacional. FASGO 2012 Sep; Vol 11, N² 2 .

7. Cordero L, Treuer SH, Landon MB, et al. Management of infants of diabetic mothers. Arch Pediatr Adolesc Med 1998; 152(3):249.

8. Moore TR. A comparison of amniotic fluid fetal pulmonary phospholipids in normal and diabetic pregnancy. Am J Obstet Gynecol 2002 Apr; 186 (4): 641-50.

9. Piper JM, Xenakis EM, Langer O. Delayed appearance of pulmonary maturation markers is associated with poor glucose control in diabetic pregnancies. Matern Fetal Med 1998; 7(3):148.

10. Piper JM. Lung maturation in diabetes in pregnancy: if and when to test. Semin Perinatol 2002 Jun; 26(3):206-9.

11. Piazze JJ, Anceschi MM, Maranghi L, et al. Fetal lung maturity in pregnancies complicated by insulin-dependent and gestational diabetes: a matched cohort study. Eur J Obstet Gynecol Reprod Biol 1999 Apr; 83(2):145-50.

12. Carlson KS, Smith BT, Post M. Insulin acts on the fibroblast to inhibit glucocorticoid stimulation of lung maturation. J Appl Physiol 1984; 57:1577-1579.

13. Guideline for the use of antenatal corticosteroids for fetal maturation. J Perinat Med 2008; 36:191-196.

14. Antenatal corticosteroids to reduce neonatal morbidity and mortality. Green-top Guideline N 7 October 2010. Royal College of Obstetricians and Gynaecologists.

15. Roberts D, Brown J, Medley N, Dalziel SR. Antenatal corticosteroids for accelerating fetal lung maturation for women at risk of preterm birth. Cochrane Database Syst Rev 2017 Mar 21; Issue 3.

16. Roberts D, Dalziel SR. Antenatal corticosteroids for accelerating fetal lung maturation for women at risk of preterm birth. Cochrane Database Syst Rev 2006; (3).

17. Brownfoot FC, Crowther CA, Middleton P. Different corticosteroids and regimens for accelerating fetal lung maturation for women at risk of preterm birth (Review). The Cochrane Library 2008; Issue 4.

18. Lee BH, Stoll BJ, McDonald SA, Higgins RD. Adverse neonatal outcomes associated with antenatal dexamethasone versus antenatal betamethasone. Pediatrics 2006;117;1503

19. Baud O, Foix-L'Helias L, Kaminski M, et al. Antenatal glucocorticoid treatment and cystic periventricular leukomalacia in very premature infants. N Engl J Med 1999; 341:1190-6.

20. Gurbuz A, Karateke A, Ozturk G et al. Is 1-hour glucose screening test reliable after a short-term administration of antenatal betamethasone? Am J Perinatol 2004 Oct; 21(7):415-20.

21. Mastrobattista JM, Patel N, Monga M. Betamethasone alteration of the one-hour glucose challenge test in pregnancy. $\mathrm{J}$ Reprod Med 2001;46:83-86.

22. JianYun $X$, Zhaoxia L, Yun $C$, et al. Changes in maternal glucose metabolism after the administration of dexamethasone for fetal lung development. Int J Endocrinol 2012; 2012.
23. Foglia LM, Deering SH, Lim E, et al. Maternal glucose levels after dexamethasone for fetal lung development in twin vs singleton pregnancies. Am J Obstet Gynecol 2008 Oct; 199 (4):380.

24. Refuerzo JS, Garg A, Rech B, et al. Continuous glucose monitoring in diabetic women following antenatal corticosteroid therapy: a pilot study. Am J Perinatol 2012 May; 29 (5):335-8.

25. Bedalov A, Balasubramanyam A. Glucocorticoid-induced ketoacidosis in gestational diabetes: sequela of the acute treatment of preterm labor. A case report. Diabetes Care 1997 Jun; 20 (6):922-4.

26. Iafusco D, Stoppoloni F, Salvia G, et al. Use of real time continuous glucose monitoring and intravenous insulin in type 1 diabetic mothers to prevent respiratory distress and hypoglycaemia in infants. BMC Pregnancy Childbirth 2008, 8:23.

27. Warburton D. Chronic hyperglycemia with secondary hyperinsulinemia inhibits the maturational response of fetal lamb lungs to cortisol. J Clin Invest 1983; 433-440.

28. Ramírez-Torres MA, Pérez-Monter SE, Espino y Sosa S, et al. Efecto de la betametasona en la glucemia de diabéticas embarazadas en riesgo de nacimiento pretérmino. Ginecol Obstet Mex 2011; 79(9):565-571.

29. Refuerzo JS, Garg A, Rech B, et al. Continuous glucose monitoring in diabetic women following antenatal corticosteroid therapy: a pilot study. Am J Perinatol 2012; 29:335-338.

30. Antenatal corticosteroids to reduce neonatal morbidity and mortality. Green-top Guideline N ${ }^{\circ} 7$ October 2010. Royal College of Obstetricians and Gynaecologists.

31. Recomendaciones para gestantes con diabetes pregestacional. Conclusiones del Consenso reunido por convocatoria del Comité de Diabetes y Embarazo de la SAD. Octubre 2009.

32. Consenso Latinoamericano de Diabetes y Embarazo. ALAD noviembre 2007.

33. Mathiesen ER, Christensen AB, Hellmuth E, et al. Insulin dose during glucocorticoid treatment for fetal lung maturation in diabetic pregnancy: test of an algorithm (correction of analgoritm). Acta Obstet Gynecol Scand 2002 Sep; 81(9):835-9.

34. Kaushal K, Gibson JM, Railton A, et al. A protocol for improved glycaemic control following corticosteroid therapy in diabetic pregnancies. Diabet Med 2003 Jan; 20 (1):73-5.

35. Dalziel SR, Liang A, Parag V, et al. Blood pressure at 6 years of age after prenatal exposure to betamethasone: follow-up results of a randomized, controlled trial. Pediatrics 2004 Sep; 114(3):e373-7.

36. Dalziel SR, Rea HH, Walker NK, et al. Long term effects of antenatal betamethasone on lung function: 30 year follow up of a randomised controlled trial. Thorax 2006 Aug; 61(8):678-83.

37. Dalziel SR, Walker NK, Parag V, et al. Cardiovascular risk factors after antenatal exposure to betamethasone: 30-year follow-up of a randomized controlled trial. Lancet 2005; 365:1856-62.

38. Dalziel SR, Lim VK, Lambert A, et al. Antenatal exposure to betamethasone: psychological functioning and health related quality of life 31 years after inclusion in randomised controlled trial. BMJ 2005 Sep 24; 331(7518):665.

39. Dalziel SR, Fenwick S, Cundy T, et al. Peak bone mass after exposure to antenatal betamethasone and prematurity: followup of a randomized controlled trial. J Bone Miner Res 2006; 21:1175-1186. 


\section{ANEXO 1 \\ Clasificación de niveles de evidencia}

$(1++)$ Metaanálisis de alta calidad, revisiones sistemáticas de ensayos controlados aleatorios o ensayos controlados aleatorios con un riesgo muy bajo de sesgo.

$(1+)$ Metaanálisis bien realizados, revisiones sistemáticas de ensayos controlados aleatorios o ensayos controlados aleatorios con bajo riesgo de sesgo.

(1-) Metaanálisis, revisiones sistemáticas de ensayos controlados aleatorios o ensayos controlados aleatorios con un alto riesgo de sesgo.

$(2++)$ Revisiones sistemáticas de alta calidad de estudios de casos y controles o estudios de cohorte o de casos y controles de alta calidad con un riesgo muy bajo de confusión, sesgo o probabilidad y una alta probabilidad de que la relación sea causal.

$(2+)$ Estudios de cohortes o casos control bien conducidos con bajo riesgo de confusión, sesgo o probabilidad y una probabilidad moderada de que la relación sea causal

(2-) Estudios de casos y controles o de cohortes con alto riesgo de confusión, sesgo o probabilidad y un riesgo significativo de que la relación no sea causal.

(3) Estudios no analíticos, por ejemplo, informes de casos, series de casos.

(4) Opinión de expertos.

\section{ANEXO 2 \\ Grados de recomendaciones}

(A) Al menos un metaanálisis, revisión sistemática o ensayo controlado aleatorizado calificado como $1++$, y directamente aplicable a la población objetivo; o una revisión sistemática de ensayos controlados aleatorios o un conjunto de evidencias que consisten principalmente en estudios calificados como 1+, directamente aplicables a la población objetivo y que demuestran la consistencia general de los resultados.

(B) Un cuerpo de evidencia que incluye estudios calificados como 2++ directamente aplicables a la población objetivo, y que demuestra la consistencia general de los resultados; o evidencia extrapolada de estudios calificados como $1++01+$.

(C) Un cuerpo de evidencia que incluye estudios calificados como 2+ directamente aplicables a la población objetivo y que demuestra la consistencia general de los resultados; o evidencia extrapolada de estudios calificados como $2++$.

(D) Nivel de evidencia 3 ó 4; o evidencia extrapolada de estudios calificados como 2+. 